\title{
Overproduction of Th2-specific chemokines in NC/Nga mice exhibiting atopic dermatitis-like lesions
}

\author{
Christian Vestergaard, ${ }^{1,2}$ Hiroyuki Yoneyama, ${ }^{1}$ Masako Murai, ${ }^{1}$ Kohichiro Nakamura, ${ }^{3}$ \\ Kunihiko Tamaki, ${ }^{3}$ Yuya Terashima, ${ }^{1}$ Toshio Imai, ${ }^{4}$ Osamu Yoshie, ${ }^{4}$ Tatsuro Irimura, ${ }^{5}$ \\ Hitoshi Mizutani, ${ }^{6}$ and Kouji Matsushima ${ }^{1}$
}

${ }^{1}$ Department of Molecular Preventive Medicine, University of Tokyo, Tokyo 113-0033, Japan

${ }^{2}$ Department of Dermatology, Marselisborg Hospital, University of Aarhus, 8000 Aarhus C, Denmark

${ }^{3}$ Department of Dermatology, School of Medicine, University of Tokyo, Tokyo 113-0033, Japan

${ }^{4}$ Department of Bacteriology, School of Medicine, Kinki University, Osaka 589-8511, Japan

${ }^{5}$ Laboratory of Cancer Biology and Molecular Immunology, Graduate School of Pharmacology, University of Tokyo, Tokyo 113-0033,

Japan

${ }^{6}$ Department of Dermatology, Mie University, Faculty of Medicine, Mie 547-8507, Japan

Address correspondence to: Kouji Matsushima, Department of Molecular Preventive Medicine, School of Medicine,

University of Tokyo, 7-3-1 Hongo, Bunkyo-ku, Tokyo 113-0033, Japan. Fax: 81-3-5684-2297; E-mail: koujim@m.u-tokyo.ac.jp.

Received for publication June 16, 1999, and accepted in revised form September 1, 1999.

\begin{abstract}
We have examined the expression of chemokines and their receptors in the atopic dermatitis-like (ADlike) lesions of NC/Nga mice. Such lesions develop when the mice are kept in conventional conditions, but not when they are kept isolated from specific pathogens. The thymus- and activation-regulated chemokine TARC is unexpectedly highly expressed in the basal epidermis of 14-week-old mice with lesions, whereas it is not expressed in the skin without lesions. Production of TARC by keratinocytes was confirmed by culturing murine keratinocytic cell line cells (PAM212) with TNF- $\alpha$, IFN- $\gamma$, or IL-1 3 . Expression of another Th2 chemokine, macrophage-derived chemokine (MDC), was observed in the skin from mice kept in both conventional and pathogen-free conditions, but expression of MDC was increased severalfold in the skin with lesions. The cellular origin of MDC was identified to be dermal dendritic cells. Infiltration of the skin by IL-4-producing T cells and mast cells, and the increase of CCR4 mRNA in the skin, coincided with the development of AD lesions. These observations indicate that TARC and MDC actively participate in the pathogenesis of AD-like lesions in NC/Nga mice and that these Th2 chemokines could be novel targets for intervention therapy of AD in humans.
\end{abstract}

J. Clin. Invest. 104:1097-1105 (1999).

\section{Introduction}

Atopic dermatitis (AD) is a clinical syndrome characterized by pruritic and eczematous skin lesions in characteristic locations, along with other major and minor clinical signs $(1,2)$. AD is generally thought to be a genetic disorder of the immune system, with patients often having a family history of $\operatorname{AD}(2,3)$. A primary ectodermal defect has been proposed to result in a disturbance of T-cell maturation (4). In addition, a reduction of ceramides in atopic skin, leading to a disrupted skin barrier has been described as an etiologic factor (5). The skin lesions are characterized by infiltrating lymphocytes, monocytes/macrophages, and fully granulated mast cells (6) and eosinophils (7). An increased number of dermal dendritic cells (DCs) and epidermal Langerhans cells has also been observed in the skin lesions of $\operatorname{AD}(8,9)$. The lymphocytes infiltrating the skin lesions of $\mathrm{AD}$ are, at least in the initiating phase of the disease, Th2-type T cells, producing IL-4, IL-5, and IL-10 (10), even though in later stages of the disease, Th1-type cytokines, such as IFN- $\gamma$, are produced in AD lesions $(2,10,11)$. IgE levels in the circulation are elevated in most, but not all, patients with $\mathrm{AD}(2)$, and this is attributed to the high production of IL-4, an inducer of IgE production (12).
The NC/Nga strain of mice originated from Japanese fancy mice and was established as an inbred strain by K. Kondo in 1957 (13). The mouse has been reported to have certain characteristics such as high susceptibility to irradiation and to anaphylactic shock induced by ovalbumin $(13,14)$. The NC/Nga mice have also been reported to develop an eczematous condition when kept in conventional surroundings but not when kept under specific pathogen-free (SPF) conditions (15). The eczema develops at the age of 8 weeks, with maximum activity at around 17 weeks with lesions characterized by edema, hemorrhage, erosion, dryness, and alopecia typically localized on the ears, back, and neck and in the facial region. The affected mice also show growth retardation. Serologically, the IgE level is markedly elevated from the age of 8 weeks, coinciding with the appearance of the skin lesions, whereas the IgG level is unaffected until the age of 12 weeks. The lesions show marked hyperkeratosis and some parakeratosis, lymphocyte infiltration with a high CD4/CD8 ratio, macrophage infiltration, and mast cell and eosinophil degranulation. IL-4 and IL-5 are both produced by mast cells in the lesions, whereas $\mathrm{CD}^{+}$cells produce only IL-4 and, in later stages, also IFN- $\gamma(15)$. The B cells of the NC/Nga mouse also have increased sensitivity to CD40 ligand and IL-4, which is proposed to be due 
to an enhanced activation of the Janus kinase 3 (JAK3). Moreover B cells of the NC/Nga mouse with dermatitis lesions show constitutive tyrosine phosphorylation of JAK3, a feature suggested to result in IgE hyperproduction in patients with $\mathrm{AD}(16)$. Treatment of the lesions of the NC/Nga mouse with tacrolimus hydrate (FK506) suppresses skin infiltration by $\mathrm{CD}^{+} \mathrm{T}$ cells, mast cells, and eosinophils, and also suppresses IL-4, IL-5, and IgE production in these mice. Steroid ointment has been found to have marginal effect (17).

Chemokines are small secreted proteins that have a major function in regulating leukocyte migration but, as has become clear over the last few years, also have a wide range of functions in nonhematopoietic tissues (18). The chemokines can be grouped, by virtue of the highly conserved cysteine residues in their sequence, into CC, CXC, C, and $\mathrm{CX} 3 \mathrm{C}$ chemokines. The receptors for the chemokines are classified based on the structure of their ligands; to date, CCR 1-9, CXCR 1-5, XCR1, and CX3CR1 have been identified (19). Recently, chemokine receptors have been reported to be expressed differentially on different subsets of T cells (20-23). CXCR3 is preferentially expressed on the Th1 subset (20), whereas CCR4 is expressed on Th2 subset (21). CCR5 is expressed on Th1 subset (22) but may also be expressed on activated T cells (23). The ligands for CCR4 are thymus- and activation-regulated chemokine (TARC) (24) and macrophage-derived chemokine (MDC) (25). TARC is a CC chemokine with a molecular mass of approximately $8 \mathrm{kDa}(26)$ and is synthesized by DCs differentiated from monocytes with GM-CSF, IL-3, and IL-4 (27). Similarly, MDC is a CC chemokine with molecular mass of 8 $\mathrm{kDa}$ synthesized by monocyte-derived macrophages (28). Both are chemotactic for a fraction of $\mathrm{CD}^{+} \mathrm{CD}^{+} 5 \mathrm{RO}^{+} \mathrm{T}$ cells polarized to produce Th2 cytokines (21). MDC and TARC share $37 \%$ identity, and are both encoded by chromosome $16(27,29)$, unlike other CC chemokines, many of which are encoded by chromosome 17 (18).

The results presented here indicate that TARC produced by basal keratinocytes and MDC produced by dermal DCs may play a significant role in recruiting Th2-type lymphocytes to the AD-like skin lesions, and that a glucocorticoid is effective in treating the lesions of the NC/Nga mouse.

\section{Methods}

Mice. SPF male and female, 8-week-old, NC/Nga mice were purchased from SLC (Tokyo, Japan). These were divided into 2 groups, one group kept in SPF environment and the other under conventional conditions. In each of the 2 groups, the mice were mated and the offspring were used for experiments. All animal experiments complied with the standards set out in the guidelines of the University of Tokyo.

Steroid treatment. Mice kept in normal conditions with fully developed skin lesions were treated with steroid ( $0.05 \%$ clobetasolepropionate) ointment (Glaxo, Tokyo, Japan), by applying the ointment to the involved region from the ears to the middle of the back. The treatment was repeated once every day for 7 days from the age of 14 weeks. As a negative control, mice of the same age with the same lesions were treated with vehicle (petroleum jelly alba) alone.

Skin biopsies. The mice were sacrificed by dislocation of the neck, upon which the hairs of the back had been removed using depilator cream (Kanebo, Tokyo, Japan). An area of approximately $1.5 \times 1.5 \mathrm{~cm}^{2}$ was excised, and the subcutaneous fat and blood vessels were carefully dissected away.

Histology. Skin biopsies were fixed with $10 \%$ formalin in neutral buffer for at least 16 hours and embedded in paraffin. Deparaffinized sections (3-5 $\mu \mathrm{m}$ thick) were stained with hematoxylin and eosin and analyzed by light microscopy.

Preparation of antibodies against MDC. A 21 amino acid-long MDC peptide from amino acids 38-58 was synthesized (PerSeptive Biosystems/Vestec Products, Tokyo, Japan) and coupled to keyhole limpet hemocyanin (KLH; Calbiochem-Novabiochem Corp., San Diego, California, USA) using maleimidobenzoyl- $N$-hydroxysuccinimideester (MB; Pierce Chemical Co., Rockford, Illinois, USA). The amino acid sequence of the fragment was QDYIRHPLPSRLVREPPWTS. The resulting KLH-MB domain-fused protein was isolated using Sephadyl S-200HR column (Pharmacia Biotech AB, Uppsala, Sweden), after which the fused protein was emulsified in CFA and used for immunization of 2 New Zealand white rabbits after 3-, 2-, 2-, and 1-week intervals. The rabbits were bled and sera obtained, and the resultant antibody specificity was tested in an ELISA. No cross-reactivity was found with other murine chemokines, including TARC, JE, and macrophage inflammatory protein-2 (MIP-2).

Immunohistochemistry. Skin biopsies were embedded in Tissue-Tek OCT compound (Miles Inc., Elkhart, Indiana, USA), snap-frozen in liquid nitrogen, and stored at $-80^{\circ} \mathrm{C}$ until use. The tissue was cut by a cryostat to $8-\mu \mathrm{m}$ sections and was thereafter incubated with the first antibody. The antibodies used were rabbit anti-mouse MDC polyantibody, a rat anti-mouse CD4 mAb (RM4-5, PharMingen, San Diego, California, USA), a rat anti-mouse CD8 mAb (53-6.7; PharMingen), a rat anti-mouse DEC-205 mAb (NLDC-145; BMA Biomedicals, Geneva, Switzerland), a rat anti-mouse MHC II (ER-TR3; BMA Biomedicals), a rat antimouse c-kit mAb (ACK45; PharMingen), a rat anti-mouse IL-4 mAb (BVD6-24G2; PharMingen), a rat anti-mouse LOM $14 \mathrm{mAb}$ (30), and a hamster anti-mouse TARC mAb (31). As negative control, a rat IgG, a hamster IgG, or a rabbit IgG was used. After staining with the first antibodies, the samples were incubated with either a horseradish peroxidase-conjugated (HRP-conjugated) goat anti-hamster IgG (Southern Biotechnology Associates Inc., Birmingham, Alabama, USA), an HRP-conjugated goat anti-rat IgG (BioSource International, Camarmillo, California, USA), or an alkaline phosphatase-conjugated donkey anti-rat IgG (Jackson ImmunoResearch Laboratories Inc., West Grove, Pennsylvania, USA). The reaction was viewed with either an AEC Substrate Kit for peroxidase or a Vector Red Alkaline Phosphatase Substrate Kit I (both from Vector Laboratories, Burlingame, California, USA).

The double immunostaining was performed as described by Matsuno et al. (32). Acetone-fixed 6- $\mu \mathrm{m}$ frozen sections were incubated with the first rat or rabbit antibodies for 1 hour. After sequential incubation with alkaline phosphatase-conjugated anti-rat or rabbit IgG for 45 minutes, labeled cells were colored red or blue with Vector Red or Vector Blue (Vector Laboratories). 
Samples were then incubated with the second rat IgG, and bound mAb's were detected with an HRP-labeled anti-rat IgG and colored brown or red with 3,3'diaminobenzidine (DAB; Wako Chemicals, Dallas, Texas, USA) substrate solution or Vector Nova Red. Slides were counterstained with Mayer's hematoxylin.

Expression of chemokines, cytokines, and chemokine receptors in the skin biopsies. Total RNA was isolated from the skin biopsies using RNAzol (Biotecx Laboratories, Houston, Texas, USA) according to the manufacturer's instruction, and reverse-transcribed into DNA using M-MLV RT 200 U/20 $\mu \mathrm{L}$ reaction volume (Sawaday, Tokyo, Japan), RNase inhibitor $40 \mathrm{U} / 20 \mu \mathrm{L}$ reaction volume (Sawaday), random primers (Promega Corp., Madison, Wisconsin, USA) 100 $\mu \mathrm{g} / 20 \mu \mathrm{L}$ reaction volume, and $3 \mu \mathrm{g}$ RNA, according to the manufacturers' instructions. One microliter of the resulting cDNA was amplified by PCR, using AmpliTaq in the buffer with $\mathrm{MgCl}_{2}$ (Perkin-Elmer Corp., Norwalk, Connecticut, USA; Roche Molecular Systems, Branchburg, New Jersey, USA). The sense primer for TARC was 5'CAGGAAGTTGGTGAGCTGGTATA-3', and the antisense primer was $5^{\prime}$-TTGTGTTCGCCTGTAGTGCATA-3'. The sense primer for MDC was 5'-TCTGATGCAGGTCCCTATGGT-3', and the antisense primer was 5'-TTATGGAGTAGCTTCTTCAC-3'. The sense primer for IL-4 was 5'-CAGCTAGTTGTCATCCTGCTCTTC-3', and the antisense primer was $5^{\prime}$-GCCGATGATCTCTCTCAAGTGA- $3^{\prime}$. The sense primer for IFN- $\gamma$ was 5 'CTCAAGTGGCATAGATGT- 3 , and the antisense primer was 5'-GAGATAATCTGGCTCTGCAGGATT-3'. The sense primer for eotaxin was 5'-AGAGCTCACAGCGCTTCTATT-3', and the antisense primer was 5'-GGTGCATCTGTTGTTGGTGATT-3'. The sense primer for CCR3 was 5'-TTGCAGGACTGGCAGCATT-3', and the antisense primer was 5 '-CCATAACGAGGAGAGGAAGAGCTA-3'. The sense primer for CCR4 was 5'-TCTACAGCGGCATCTTCTTCAT-3', and the antisense primer was 5'CAGTACGTGTGGTTGTGCTCTG-3'. The sense primer for CCR5 was 5'-CATCGATTATGGTATGTCAGCACC-3', and the antisense primer was 5'-CAGAATGGTAGTGTGAGCAGGAA-3'. The sense primer for CXCR3 was 5'-ATCAGCGCTTCAATGCCAC-3' ${ }^{\prime}$, and the antisense primer was 5'-TGGCTTTCTCGACCACAGTT-3'. The sense primer for GAPDH was 5'-AGTATGACTCCACTCACGGCAA-3', and the antisense primer was 5'-TCTCGCTCCTGGAAGATGGT-3'. The reaction products were viewed on a $3 \%$ polyacrylamide gel with ethidium-bromide.

Quantitative PCR. To quantitate the mRNA in the skin of the aforementioned cytokines, chemokines, and chemokine receptors, quantitative PCR using the ABI 7700 sequencer detection system (Perkin-Elmer Applied Biosystems, Foster City, California, USA) was performed. The reaction mixture was prepared according to the manufacturer's instructions to yield final concentrations of 1 $\times$ PCR buffer A; $200 \mu \mathrm{M}$ dATP, dGTP, and dCTP each; $400 \mu \mathrm{M}$ dUTP; 4 mM MgCl2; 1.25 U AmpliTaq Gold DNA polymerase; and $200 \mu \mathrm{M}$ of each primer. To the separate reactions were also added the following target hybridization probes $(100 \mu \mathrm{M})$ for specific detection. For MDC, the probe 5'-CCAATGTGGAAGACAGTATCTGCTGCCA$3^{\prime}$ was added. For CCR5, the probe 5'-TACCTGCTCAACCTGGCCATCTCTGA-3' was added. For GAPDH, the probe 5'-AACGGCACAGTCAAGGCCGAGAAT-3' was added. The probe was labeled with a reporter fluorescent dye, FAM (6-carboxyflourescein), at the $5^{\prime}$-end. The thermal cycling conditions were set to $50^{\circ} \mathrm{C}$ for 2 minutes and $95^{\circ} \mathrm{C}$ for 10 minutes, followed by 50 cycles of amplification at $95^{\circ} \mathrm{C}$ for 15 seconds and $55^{\circ} \mathrm{C}$ for 1.5 minutes for denaturing and annealing/extension, respectively. To compare mRNA expression, the results were compared as relative values using GAPDH as an internal reference and the samples from mice kept in SPF conditions as overall reference. The relative value was calculated after the following formula: relative expression $=2\left[-\left(\Sigma V_{s} / N s-\Sigma G A P D H s / N s\right)-\right.$ $(\Sigma V r / N r-\Sigma G A P D H r / N r)]$, where $V s$ denotes the ABI Prism signal from a given sample, $N s$ is the number of samples, GAPDHs is the ABI Prism signal for GAPDH in the same sample, $V r$ is the ABI Prism signal for the same sample but in the overall reference sample, and GAPDHr is the ABI Prism GAPDH signal in the reference sample. The number of samples was $n=3$ in each group.

TARC production by murine keratinocytes in vitro. Murine keratinocytes (PAM 212; American Type Culture Collection, Rockville, Maryland, USA) were cultured in RPMI1640 (GIBCO BRL, Rockville, Maryland, USA) and 10\% FCS at a concentration of $4 \times 10^{4}$ cells $/ \mathrm{mL}$ in $1-\mathrm{mL}$ wells for 4 days and were stimulated with 1 of the following cytokines; $10 \mathrm{ng} / \mathrm{mL}$ TNF- $\alpha$ (R\&D Systems Inc., Minneapolis, Minnesota, USA); $100 \mathrm{ng} / \mathrm{mL}$ TNF- $\alpha$ and 10 ng/mL GM-CSF (Genzyme Japan, Tokyo, Japan); 1.0 $\mathrm{ng} / \mathrm{mL}$ IFN- $\gamma$ (R\&D Systems Inc.); $100 \mathrm{ng} / \mathrm{mL}$ IFN- $\gamma$ and $10 \mathrm{pg} / \mathrm{mL}$ IL-1 $\beta$ (Genzyme Japan); and $1 \mathrm{ng} / \mathrm{mL}$ IL- $1 \beta 1$ and $100 \mathrm{pg} / \mathrm{mL}$ or $1 \mathrm{ng} / \mathrm{mL}$ IL-4 (Becton Dickinson and Co., Franklin Lakes, New Jersey, USA). All experiments were carried out in triplicate, except for the IL-4 experiment, which was carried out only in duplicate. After 4 days, the supernatants of the cell cultures were isolated and stored at $-80^{\circ} \mathrm{C}$ until use. To assess the production of TARC, ELISA was performed. Briefly, a 96-well plate (F96; MaxiSorp, NUNC A/S, Roskilde, Denmark) was plated with hamster anti-mouse TARC polyantibody and blocked with $1 \mathrm{mg} / \mathrm{mL}$ BSA in PBS, after which the supernatants were added. The second antibody was a biotinylated rabbit anti-TARC, and after incubation, the result was viewed with HRP-streptavidin according to the manufacturer's instructions (Vector Laboratories) and read with an Emax ELISA reader at 450-nm wavelength.

\section{Results}

The offspring of the NC/Nga mice were kept in 2 groups: 1 group under SPF conditions, and another group under conventional conditions. The mice kept under conventional conditions started to scratch themselves at the age of 8 weeks, at which time the skin became dry and scaly. Within the next 2 weeks, the mice developed lesions located on the ears and neck and in the face. The lesions on the back were located from the ears and down to the middle of the back and reached forward to the posterior axillary line. The lesions consisted of excoriations and nodular infiltrations that could be palpated through the skin (Figure 1a). The nodular lesions were absent, and only excoriations could be found on the ears and in the face. The mice kept in SPF conditions did not develop lesions (Figure 1b). Pregnant female mice kept under conventional conditions developed less-severe lesions.

The cellular composition of the infiltrate in the skin lesions. 
Paraffin-embedded sections of both lesional (14-week-old mice kept in conventional conditions) and nonlesional (14-week-old mice kept in SPF conditions) skins were prepared. The lesional skin showed hyperkeratosis, acanthosis, and parakeratosis in the epidermis (Figure 1d) in contrast to the normal nonlesional skin (Figure 1e). The lesional skin also showed a heavy infiltrate in the dermis with dense connective tissue, whereas the nonlesional skin did not show any infiltration. The cells infiltrating the dermis were identified by immunostaining of frozen sections. The lymphocytes invading the lesional skin were mainly $\mathrm{CD}^{+}$cells (Figure $2 \mathrm{a}$ ), with a few $\mathrm{CD}^{+}$cells (data not shown). The dermal macrophages in lesional (Figure 2b) and nonlesional (Figure 2c) skin were identified using an antibody against MMGL (LOM14), and the number of macrophages was increased in the lesional skin (Table 1) when compared with the nonlesional skin. Non-lymphoid dendritic cell-positive (NLDC-positive) cells were largely observed to locate both in the dermis and in the epidermis (Figure 2d). The NLDC-positive cells in the epidermis were considered to be Langerhans cells. Cells in the dermis and in the epidermis equally stained for MHC II (Figure 2e), although the number of MHC II-positive cells in the dermis were substantially higher than NLDC-positive cells (Table 1). The double immunostaining, in addition to similar morphology and distribution, revealed that the cells positive for NLDC in the dermis were also positive for MHC II (Figure 2f) and were identified as dermal DCs. The remaining MHC II-positive cells had similar morphology and distribution with LOM14-positive cells and were identified as macrophages. Last, very large populations of big amorphous cells positive for c-kit in the dermis of both lesional (Figure $2 \mathrm{~g}$ ) and, in smaller number (Table 1), nonlesional skin (Figure $2 \mathrm{~h}$ ) were considered to be mast cells. In addition, a c-kit-positive cell population of dendritic-shaped cells were found in the epidermis
(Figure 2g) of the lesional skin, but not in the nonlesional biopsies. Cells in the skin expressing c-kit have been described as being either mast cells (33) and their progenitors (34) or melanocytes (35). To identify the c-kit-positive cells, toluidine blue staining for mast cells was performed. The dendritic-shaped cells in the epidermis were not stained with toluidine blue, suggesting that they were melanocytes (data not shown).

Increased expression of TARC in the skin with aging and exacerbation of the lesions. Total RNA was isolated from skin biopsies obtained from mice at ages 6, 8 and 12 weeks, and RT-PCR was performed as described in Methods. In the skin from mice kept in conventional conditions, TARC was already weakly expressed at the 6 weeks of age, and at the same level at 8 weeks. At 12 weeks of age, when the skin lesions became conspicuous, the expression of TARC was markedly increased (Figure 3a). In the skin from mice kept in SPF conditions, no TARC expression could be detected (Figure 3a). This was also the case when quantitative RT-PCR was performed (data not shown, as a relative value cannot be expressed). Using an anti-TARC $\mathrm{mAb}$, the production of TARC was localized mainly to the basal layer of the epidermis (Figure 2i). Furthermore, in the skin of mice kept under SPF conditions, no TARC production could be observed by immunostaining (Figure 2j).

Production of TARC by keratinocytes in vitro. When murine keratinocytic cell line (PAM 212) cells were cultured and stimulated with various cytokines, production of TARC could be induced by TNF- $\alpha$, IFN- $\gamma$, and IL-1 $\beta$ (Figure 4) as measured by ELISA. The production of TARC induced by $10 \mathrm{ng} / \mathrm{mL}$ TNF- $\alpha$ was significantly higher than the production induced by IFN- $\gamma$ and IL- $1 \beta$ at all concentrations $(P<0.05)$, whereas there was no significant difference in the production of TARC induced by $100 \mathrm{ng} / \mathrm{mL}$ TNF- $\alpha$ and by IFN- $\gamma$ and IL- $1 \beta$ at all concentrations. Nei-

\footnotetext{
Figure 1

The appearance of skin lesions on mice 14 weeks old, kept in conventional conditions (a); 14 weeks old, kept in SPF conditions (b); and 15 weeks old, kept in conventional conditions but treated with steroid ointment for 7 days from 14 weeks of age (c). The hair was removed using depilator cream as described in Methods. Hematoxylin-eosin staining of paraffin-embedded sections (3-5 $\mu \mathrm{m}$ thick) from skin biopsies from 14-week-old mice kept in conventional conditions (d) and SPF conditions (e), and from 15-week-old mice kept in conventional conditions and treated with steroid for 7 days (f). The results shown are representative of 3 mice in each group. Note the thickening of the epidermis and keratin layer in $\mathbf{d}$ compared with $\mathbf{e}$, and the nuclei of the keratinocytes being visible into the keratin layer. Also note the remarkable effect of the steroid ointment in suppressing both the infiltration in the dermis and the growth of the epidermis. $\times 100$.
}

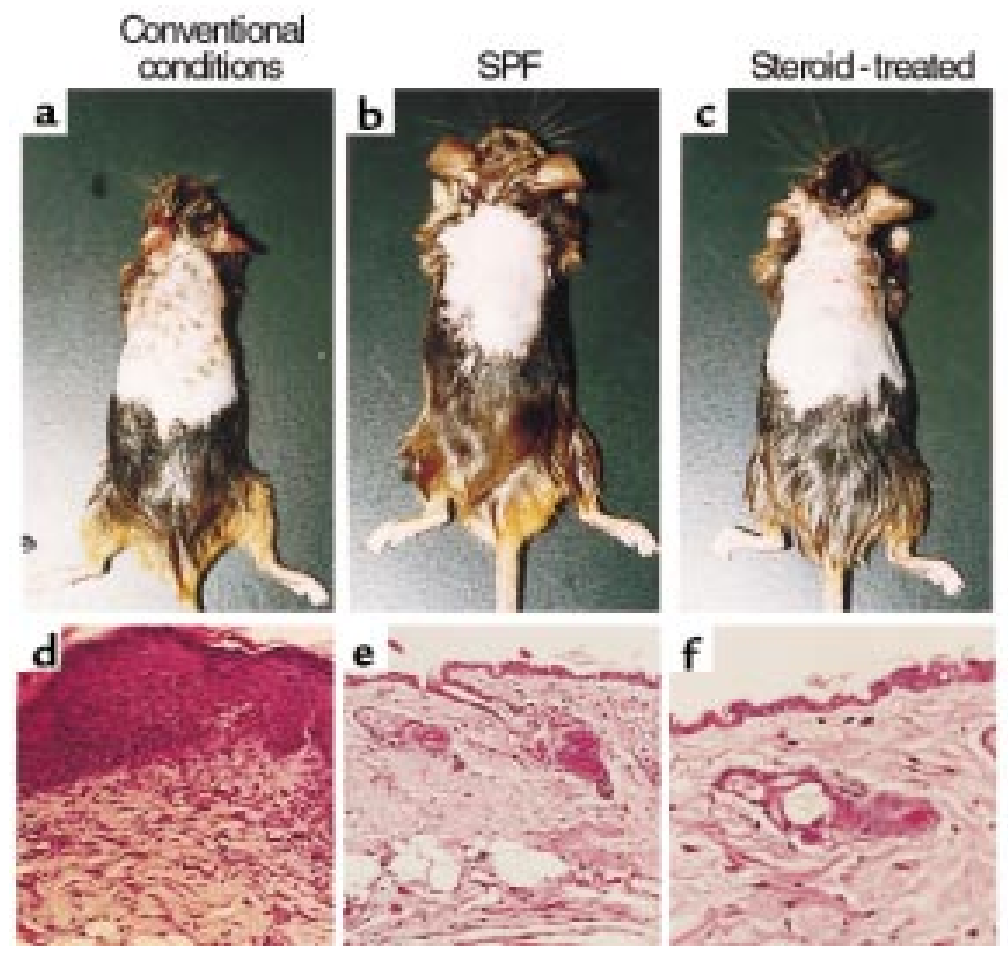



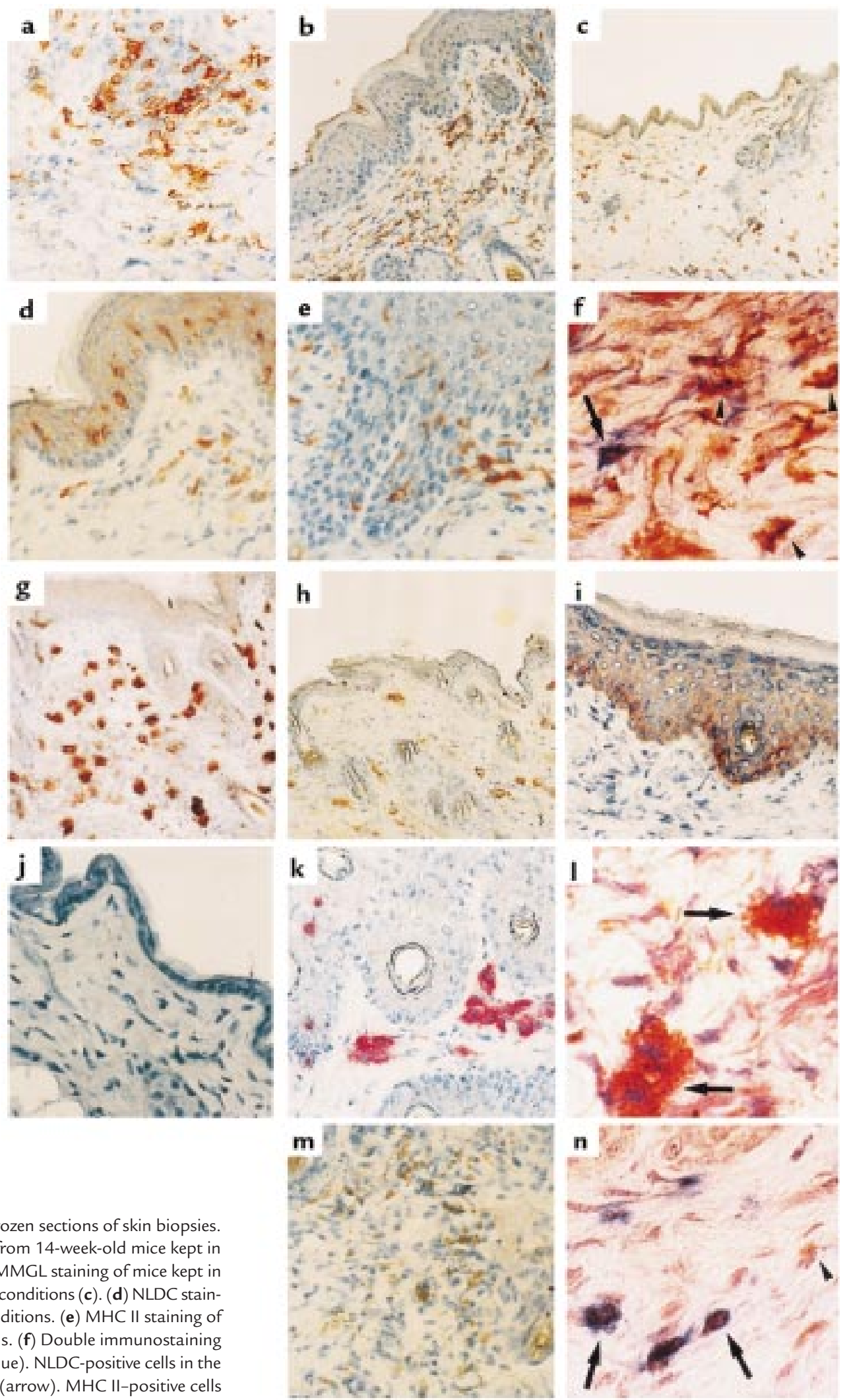

Immunohistochemical staining of frozen sections of skin biopsies. (a) CD4 staining of biopsies taken from 14-week-old mice kept in conventional conditions. ( $\mathbf{b}$ and $\mathbf{c}$ ) MMGL staining of mice kept in conventional conditions (b) and SPF conditions (c). (d) NLDC staining of mice kept in conventional conditions. (e) MHC II staining of mice kept in conventional conditions. (f) Double immunostaining for MHC II (dark red) and NLDC (blue). NLDC-positive cells in the dermis are also positive for MHC II (arrow). MHC II-positive cells (arrowheads) are identified as macrophages. ( $\mathbf{g}$ and $\mathbf{h}$ ) c-kit staining of skin biopsies from mice kept in conventional conditions $(\mathbf{g})$ and SPF conditions (h). ( $\mathbf{i}$ and $\mathbf{j})$ TARC staining of skin from mice kept in conventional conditions (i) and SPF conditions (j). (k) MDC staining of mice kept in conventional conditions. (I) Double immunostaining for NLDC (brown) and MDC (red). Approximately $80 \%$ of NLDC-positive cells are also positive for MDC (arrow). MDC-producing NLDC-positive cells (double-positive cells, compared with k) are identified as dermal DCs. ( $\mathbf{m}$ ) IL-4 staining of mice kept in conventional conditions. (n) Double immunostaining for CD4 (blue) and IL-4 (dark red). Most of $\mathrm{CD}^{+}$(colored blue with cell surface) cells are positive for IL-4 (colored dark red with cytoplasm; arrow). Some cells show single positive for IL-4 (arrowheads), which are identified as mast cells. Original magnifications: $\times 100(\mathbf{a}-\mathbf{d}, \mathbf{g}-\mathbf{j}), \times 200(\mathbf{e}, \mathbf{f}, \mathbf{k}, \mathbf{m}$, and $\mathbf{n}), \times 400(\mathbf{I})$. 


\section{Figure 3}

(a) RT-PCR of mRNA isolated from skin biopsies taken at the times indicated. Left: expression of IFN- $\gamma$, MDC, TARC, and GAPDH from mice kept in SPF conditions at the age of 6, 8, and 12 weeks. Right: expression of the mRNA at the same time points, but from mice kept in normal conditions. The results are representative of 6,4 , and 6 mice in SPF conditions and 6, 4 and 6 mice in normal conditions; the ratio of males to females was 1:1. (b) RT-PCR performed on skin from 14-weekold mice kept in normal conditions (top) and SPF conditions (middle), and from 15-week-old mice kept in normal conditions and treated with steroid from the age of 14 weeks (bottom). The $x$ axis shows the gene expression examined. The results are representative of 6,6 , and 3 independent experiments, respectively. a

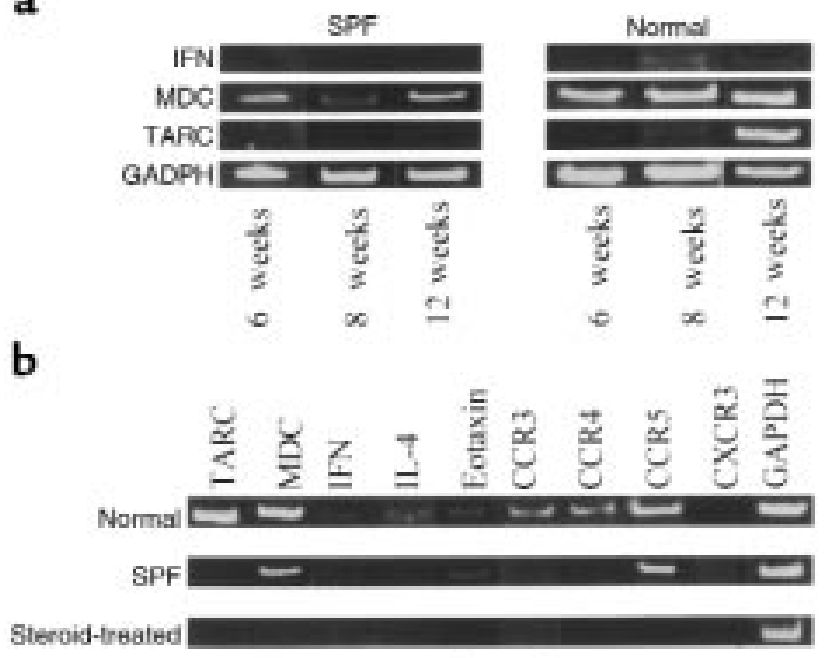

b

14 weeks

expressed in lesional skin only (Figure 3b), and the cells producing IL-4 were identified to be mast cells and lymphocytes (Figure 2, $m$ and $n$ ). Eotaxin was found by RTPCR to be expressed in both lesional and nonlesional skin. The receptors CCR3 and CCR5 were expressed in both lesional and nonlesional skin (Figure $3 \mathrm{~b}$ ), but the level of CCR5 expression was 10 times higher in lesional skin than in nonlesional (Figure 5). CXCR3 was not expressed in lesional or nonlesional skin.

Effect of topical steroid treatment. When 14-week-old mice kept in normal conditions were treated with steroid ointment for 7 days $(n=3)$, the lesions on the back showed extensive regression (Figure 1c). The injury on the ears and the back appeared to heal, but the skin was observed to be very thin. The number of $\mathrm{CD}^{+}$cells in the skin decreased below normal levels (Table 1), and that of $\mathrm{CD}^{+}$cells returned to normal levels (Table 1 ). The number of macrophages and c-kit-positive cells decreased compared with untreated lesional skin, but did not return to normal levels. The number of NLDC-positive cells decreased in the epidermis and dermis compared with nonlesional skin, as did the number of MHC II-positive cells in steroid-treated skin (Table 1). The expression of cytokines, chemokines, and chemokine

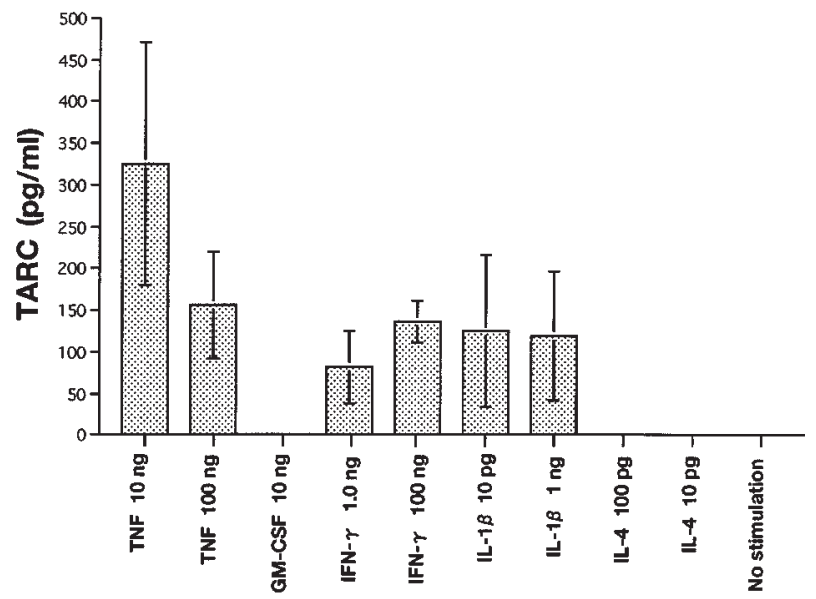

\section{Figure 4}

The results of TARC ELISA performed on the culture supernatants from keratinocyte cell cultures after 4 days of culturing. The $x$ axis indicates the stimuli added to the cultures, and the $y$ axis is the amount of TARC in the culture supernatants from the cell cultures. The results are the average values obtained from 3 different experiments each value determined in doublets. Error bars indicate SD. 
receptors were assessed using both RT-PCR (Figure 3b) and quantitative RT-PCR (data not shown). In neither case could any expression of the mRNA of the chemokines, cytokines, and chemokine receptors be detected. The mice treated with vehicle showed no difference from the 14-week-old mice kept in conventional conditions (data not shown).

\section{Discussion}

The NC/Nga mouse has been proposed as a model of human $\mathrm{AD}$. The skin lesions that developed in these mice when kept under conventional conditions resemble those of human $\mathrm{AD}$, with marked $\mathrm{CD} 4^{+}$lymphocyte infiltration accompanied by macrophages, eosinophils, and mast cells (15). This mouse strain has also been shown to have an increase in serum IgE levels as in human patients with AD, which has been shown to be due to an enhanced phosphorylation of JAK3 in these mice and probably also in human patients with $\mathrm{AD}(16)$. The eliciting factor of the dermatitis is not known, but when BALB/c mice were kept under conventional conditions with the NC/Nga mice, they did not develop any lesions, indicating that a genetic factor in addition to an environmental factor is responsible for the development of the dermatitis (15). AD has been described to be a Th2-type disease, at least in the initiating phase, as lymphocytes invading the skin mainly produce IL-4, IL-5, and IL-10 (10). The chemokine receptor predominantly expressed on Th2-type human T cells is CCR4 $(21,23)$, and the ligands for this receptor are TARC (25) and MDC (24). TARC/MDC-CCR4 system has been shown to be involved in Th2-mediated disease process, even in murine models $(31,36)$. Production of IL-4 and IL-5 in skin lesions of the $\mathrm{NC}$ /Nga mice has been described (15), and the production of IL-4 in mast cells and lymphocytes in the dermis of lesional skin is also demonstrated in that study.

On the basis of these results, the pathogenesis of the skin lesions in the NC/Nga mice has been postulated to be dictated by Th2-type cytokines, and the expression of the ligands for CCR4 in these mice was examined in this study. Overexpression of TARC by the basal keratinocytes as the lesions develops in the skin of the $\mathrm{NC} / \mathrm{Nga}$ mice suggests that TARC plays a key role in the pathogenesis of the lesions. When murine keratinocytic cell line cells were cultured in the presence of several inflammatory cytokines, TNF- $\alpha$ was the most potent inducer of TARC. Induction of TARC by Th1 cytokine IFN- $\gamma$ in keratinocytes was rather unexpected but is consistent with high expression of TARC in the skin of the NC/Nga mice at a time when concomitant expression of IFN- $\gamma$ was observed. On the other hand, a Th2-type cytokine IL-4 did not induce TARC production. TNF- $\alpha$ has been reported to be upregulated in mast cells in the skin of AD lesions (37) and to induce expression of adhesion molecules in the endothelium in lesional AD skin (38), suggesting a dual role for TNF- $\alpha$ as an inducer of chemokines and adhesion molecules, thereby acting on 2 different molecules in the chemotactic process. Keratinocytes are known to produce several cytokines, such as IL-1, IL-6, and IL-8 (39), but this is the first time that TARC has been produced by keratinocytes. This finding suggests that TARC produced by activated keratinocytes could be an early

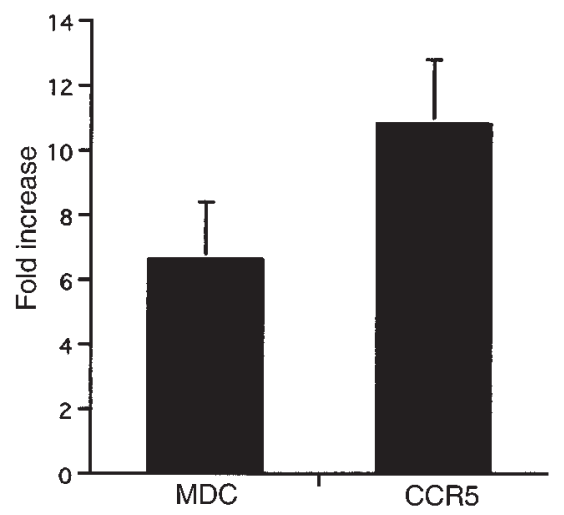

\section{Figure 5}

Quantitative RT-PCR for MDC and CCR5. The ratios of expression for MDC and CCR5 between mice kept in normal and SPF condition were calculated using GADPH as an internal reference and the expression in the SPF skin as baseline. The results were calculated from 3 independent experiments. Error bars indicate SD.

inducer of the Th2 response by attracting CCR4 $4^{+}$Th2 T lymphocytes to the lesional skin.

The second ligand using the chemokine receptor CCR4 is MDC, and we found a constitutive expression of MDC in both lesional and nonlesional skin in mice ages 6-14 weeks. Using anti-MDC polyantibody, the cells producing MDC were identified to be large round or spindle-shaped cells. The quantitative RT-PCR showed significant difference in the expression of MDC. However, the distribution of the MDC-positive cells differed in lesional and nonlesional skin, with cells lying deeper in the dermis and the subcutaneous tissue in the nonlesional skin compared to the lesional skin. These results indicate a more complex role of MDC than TARC in the pathogenesis of the $\mathrm{AD}$-like skin lesions in the NC/Nga mice. MDC may, under SPF conditions, act as a homeostatic chemokine, regulating the normal leukocyte trafficking through the skin. The importance of TARC and MDC in the pathogenesis of the ADlike disease in the NC/Nga mice is the enhanced expression of CCR4 mRNA in the lesional skin that was not expressed at all in the nonlesional skin. This finding also strengthen the evidence that the skin disease is subject to a Th2-type cytokine response. To establish whether TARC and MDC are selectively involved in Th2-mediated skin disease, further investigations in other eczematous lesions induced by contact allergens and infectious agents are required.

\section{Table 1}

The cellular composition of the infiltrate in the skin lesions.

\begin{tabular}{lcccccc} 
& \multicolumn{3}{c}{$\begin{array}{l}\text { Marker } \\
\text { Conventional conditions }\end{array}$} & \multicolumn{2}{c}{ SPF } & \multicolumn{2}{c}{ Steroid Treatment } \\
CD4 & 37 & $( \pm 2.79)$ & 13.2 & $( \pm 3.89)$ & 3.2 & $( \pm 1.08)$ \\
CD8 & 10.4 & $( \pm 1.95)$ & 0.4 & $( \pm 0.48)$ & 1 & $( \pm 0.77)$ \\
Dermal Mø & 66.8 & $( \pm 7.11)$ & 23.8 & $( \pm 3.4)$ & 45.1 & $( \pm 5.28)$ \\
C-kit & 40.4 & $( \pm 9.15)$ & 16.6 & $( \pm 4.96)$ & 27.4 & $( \pm 3.72)$ \\
NLDC & 17.8 & $( \pm 3.54)$ & 4.8 & $( \pm 1.25)$ & 2.3 & $( \pm 1.29)$ \\
MHCII & 72.2 & $( \pm 3.76)$ & 30.8 & $( \pm 2.27)$ & 4 & $( \pm 1.34)$
\end{tabular}

The number of cells counted in immunohistochemical stainings of $8 \mathrm{~mm}$ thick cryosections of skin biopsies from 14 weeks old mice kept in normal conditions, SPF conditions and 15 weeks old mice kept in normal conditions and treated with steroid for 1 week. The left side column describes which cell has been counted. The cells were counted in 5 consecutive fields at $\times 400$ magnification in sections from two different mice. The numbers in the parentheses are the standard deviations. 
Eotaxin is known as a potent chemoattractant for eosinophils (40) and a ligand for CCR3 in both humans (41) and mice (42). In our study, eotaxin was expressed in both lesional and nonlesional skin, but the receptor was only expressed in lesional skin. This is in line with the description of eosinophils as a cell infiltrating the skin of $\mathrm{AD}$ lesions $(7,15)$.

Regarding the cellular composition of the dermal infiltrate in the lesional skin, our results confirm those already observed (15), with the infiltrate consisting of lymphocytes with a high $\mathrm{CD} 4^{+} / \mathrm{CD}^{+}$ratio and a high number of macrophages and mast cells. In addition, the number of NLDC- and MHC II-positive cells were increased in the skin lesions, but with a much higher number of MHC II-positive cells. Some of the NLDCpositive cells were also positive for MHC II and were, in all likelihood, dermal DCs, but the remaining MHC II-positive cells were probably macrophages based on their morphology and distribution of MMGL-positive cells. In addition, these NLDC- and MHC II-positive dermal DCs were revealed to be the source of MDC in the lesional skin by double immunostaining. In the epidermis, a large number of NLDC- and MHC II-positive cells were found; these were likely to be Langerhans cells.

Steroid treatment for 1 week had a remarkable ameliorating effect on the lesions of the mice kept in conventional conditions: the lesions almost disappeared. But such treatment also had adverse effect, as the treated skin in some areas became very thin. The regression of the acanthosis and hyperkeratosis may be due to removal of the pruritus, which in turn stops the mouse from scratching itself, thereby interrupting the lichinifying process, rather than being a direct effect of steroids on the epidermis. Microscopically, the infiltration was markedly reduced, leaving only very few $\mathrm{CD}^{+}$cells and almost no $\mathrm{CD}^{+}$cells. On the other hand, the number of dermal macrophages was still high, as was the number of mast cells, which may be due either to these particular cells' lower sensitivity to steroid or to a longer turnover time in the skin. The result presented here is apparently in disagreement with results reported earlier (17), but it may be because of a larger amount of topical steroid used in this study.

The results presented here support the previous reports on the $\mathrm{NC} / \mathrm{Nga}$ mouse as a model for human AD. Interestingly, the levels TARC produced mainly by the keratinocytes of basal layer in the skin correlated with the severity of the skin lesions, whereas MDC was produced by dermal DCs in both lesional and nonlesional skin, although the level of MDC production increased in lesional skin, as did the number of MDCproducing cells. Surprisingly, TARC, a Th2 chemokine, could be induced in keratinocytic cell line cells by IFN- $\gamma$, a Th1 cytokine, which, although consistent with the results observed in the skin of the NC/Nga mouse and also in human patients with $\mathrm{AD}(11)$, is a unique augmentation of 1 Th-type response by the other. Further investigations of these observations in humans are needed, but this might be an important clue in investigating the complexity of the pathogenesis of AD in humans, as this shows a direct connection between the Th1 and Th2 response in the skin.

\section{Acknowledgments}

We are grateful to Joost J. Oppenheim (National Cancer Institute, Frederick, Maryland, USA) for the pre-review of the manuscript. This study was supported in part by a grant from Core Research and Revolutional Science and Technology, Japan Science and Technology Corporation.

1. Hanifin, J., and Rajka, G. 1980. Diagnostic features of atopic dermatitis. Acta Derm. Venereol. Suppl. (Stockh.) 92:44-47.

2. Rudikoff, D., and Lebwohl, M. 1998. Atopic dermatitis. Lancet. 351:1715-1721.

3. Cooper, K.D. 1994. Atopic dermatitis: recent trends in pathogenesis and therapy [see comments]. J. Invest. Dermatol. 102:128-137.

4. Thestrup-Pedersen, K., Ellingsen, A.R., Olesen, A.B., Lund, M., and Kaltoft, K. 1997. Atopic dermatitis may be a genetically determined dysmaturation of ectodermal tissue, resulting in disturbed T-lymphocyte maturation. A hypothesis. Acta Derm. Venereol. Suppl. (Stockh.) 77:20-21.

5. Imokawa, G., et al. 1991. Decreased level of ceramides in stratum corneum of atopic dermatitis: an etiologic factor in atopic dry skin? J. Invest. Dermatol. 96:523-526.

6. Soter, N.A. 1989. Morphology of atopic eczema. Allergy. 44:16-19.

7. Uehara, M., Izukura, R., and Sawai, T. 1990. Blood eosinophilia in atopic dermatitis. Clin. Exp. Dermatol. 15:264-266.

8. Taylor, R.S., Baadsgaard, O., Hammerberg, C., and Cooper, K.D. 1991. Hyperstimulatory CD1a+CD1b+CD36+ Langerhans cells are responsible for increased autologous $\mathrm{T}$ lymphocyte reactivity to lesional epidermal cells of patients with atopic dermatitis. J. Immunol. 147:3794-3802.

9. Bos, J.D., van Garderen, I.D., Krieg, S.R., and Poulter, L.W. 1986. Different in situ distribution patterns of dendritic cells having Langerhans $(\mathrm{T} 6+)$ and interdigitating (RFD1+) cell immunophenotype in psoriasis, atopic dermatitis, and other inflammatory dermatoses. J. Invest. Dermatol. 87:358-361.

10. Grewe, M., et al. 1998. A role for Th1 and Th2 cells in the immunopathogenesis of atopic dermatitis. Immunol. Today. 19:359-361.

11. Grewe, M., Gyufko, K., Schopf, E., and Krutmann, J. 1994. Lesional expression of interferon-gamma in atopic eczema. Lancet. 343:25-26.

12. Pene, J., et al. 1988. IgE production by normal human lymphocytes is induced by interleukin 4 and suppressed by interferons gamma and alpha and prostaglandin E2. Proc. Natl. Acad. Sci. USA. 85:6880-6884.

13. Kondo, K., Nagami, T., and Tadokoro, S. 1969. Differences in haematopoeitic death among inbred strains of mice. In Comparative cellular and species radiosensitivity. P. Bond and S. Sugawara, editors. Igakushoin. Tokyo, Japan. p. 20.

14. Festing, M. 1996. Origins and characteristics of inbred strains of mice. In Genetic variants and strains of the laboratory mouse. M. Lyon, S. Rastan, and S. Brown, editors. Oxford University Press. New York, NY. p. 1537.

15. Matsuda, H., et al. 1997. Development of atopic dermatitis-like skin lesion with IgE hyperproduction in $\mathrm{NC} / \mathrm{Nga}$ mice. Int. Immunol. 9:461-466.

16. Matsumoto, M., et al. 1999. IgE hyperproduction through enhanced tyrosine phosphorylation of Janus kinase 3 in NC/Nga mice, a model for human atopic dermatitis. J. Immunol. 162:1056-1063.

17. Hiroi, J., et al. 1998. Effect of tacrolimus hydrate (FK506) ointment on spontaneous dermatitis in NC/Nga mice. Jpn. J. Pharmacol. 76:175-183.

18. Rollins, B.J. 1997. Chemokines. Blood. 90:909-928.

19. Luster, A.D. 1998. Chemokines: chemotactic cytokines that mediate inflammation. N. Engl. J. Med. 338:436-445.

20. Bonecchi, R., et al. 1998. Differential expression of chemokine receptors and chemotactic responsiveness of type $1 \mathrm{~T}$ helper cells (Th1s) and Th2s. J. Exp. Med. 187:129-134.

21. Imai, T., et al. 1999. Selective recruitment of CCR4-bearing Th2 cells toward antigen-presenting cells by the CC chemokine thymus and activation-regulated chemokine and macrophage-derived chemokine. Int. Immunol. 11:81-88.

22. Loetscher, P., et al. 1998. CCR5 is characteristic of Th1 lymphocytes [letter]. Nature. 391:344-345.

23. Sallusto, F., Lanzavecchia, A., and Mackay, C. 1998. Chemokine and chemokine receptors in T-cell priming and Th1/Th2-mediated response. Immunol. Today. 19:568-574.

24. Imai, T., et al. 1997. The T cell-directed CC chemokine TARC is a highly specific biological ligand for CC chemokine receptor 4. J. Biol. Chem. 272:15036-15042.

25. Imai, T., et al. 1998. Macrophage-derived chemokine is a functional ligand for the CC chemokine receptor 4. J. Biol. Chem. 273:1764-1768.

26. Imai, T., et al. 1996. Molecular cloning of a novel T cell-directed CC chemokine expressed in thymus by signal sequence trap using EpsteinBarr virus vector. J. Biol. Chem. 271:21514-21521.

27. Hashimoto, S., Suzuki, T., Dong, H., Yamazaki, N., and Matsushima, K. 1999. Serial analysis of gene expression in human monocytes and macrophages. Blood. 94:837-844. 
28. Godiska, R., et al. 1997. Human macrophage-derived chemokine (MDC), a novel chemoattractant for monocytes, monocyte-derived dendritic cells, and natural killer cells. J. Exp. Med. 185:1595-1604.

29. Nomiyama, H., et al. 1997. Assignment of the human CC chemokine gene TARC (SCYA17) to chromosome 16q13. Genomics. 40:211-213.

30. Kimura, T., Imai, Y., and Irimura, T. 1995. Calcium-dependent conformation of a mouse macrophage calcium-type lectin. Carbohydrate binding activity is stabilized by an antibody specific for a calcium-dependent epitope. J. Biol. Chem. 270:16056-16062.

31. Yoneyama, H., et al. 1998. Pivotal role of TARC, a CC chemokine, in bacteria-induced fulminant hepatic failure in mice. J. Clin. Invest. 102:1933-1941.

32. Matsuno, K., Ezaki, T., Kudo, S., and Uehara, Y. 1996. A life stage of particle-laden rat dendritic cells in vivo: their terminal division, active phagocytosis, and translocation from the liver to the draining lymph. $J$. Exp. Med. 183:1865-1878.

33. Ghannadan, M., et al. 1998. Phenotypic characterization of human skin mast cells by combined staining with toluidine blue and CD antibodies. J. Invest. Dermatol. 111:689-695.

34. Rodewald, H.R., Dessing, M., Dvorak, A.M., and Galli, S.J. 1996. Identification of a committed precursor for the mast cell lineage. Science. 271:818-822.

35. Grabbe, J., Welker, P., Dippel, E., and Czarnetzki, B.M. 1994. Stem cell factor, a novel cutaneous growth factor for mast cells and melanocytes.
Arch. Dermatol. Res. 287:78-84.

36. Gonzalo, J.-A., et al. 1999. Mouse monocyte-derived chemokines is involved in airway hyperreactivity and lung inflammation. J. Immunol. 163:403-411

37. Ackermann, L., and Harvima, I.T. 1998. Mast cells of psoriatic and atopic dermatitis skin are positive for TNF-alpha and their degranulation is associated with expression of ICAM-1 in the epidermis. Arch. Dermatol. Res. 290:353-359.

38. de Vries, I.J., et al. 1998. Adhesion molecule expression on skin endothelia in atopic dermatitis: effects of TNF-alpha and IL-4. J. Allergy Clin. Immunol. 102:461-468.

39. Teunissen, M.B., Koomen, C.W., de Waal Malefyt, R., Wierenga, E.A., and Bos, J.D. 1998. Interleukin-17 and interferon-gamma synergize in the enhancement of proinflammatory cytokine production by human keratinocytes. J. Invest. Dermatol. 111:645-649.

40. Kitaura, M., et al. 1996. Molecular cloning of human eotaxin, an eosinophil-selective CC chemokine, and identification of a specific eosinophil eotaxin receptor, CC chemokine receptor 3. J. Biol. Chem. 271:7725-7730.

41. Daugherty, B.L., et al. 1996. Cloning, expression, and characterization of the human eosinophil eotaxin receptor. J. Exp. Med. 183:2349-2354.

42. Gao, J.L., et al. 1996. Identification of a mouse eosinophil receptor for the CC chemokine eotaxin. Biochem. Biophys. Res. Commun. 223:679-684 